\title{
BinCam: Designing for Engagement with Facebook for Behavior Change
}

\author{
Rob Comber ${ }^{1}$, Anja Thieme ${ }^{1}$, Ashur Rafiev ${ }^{1}$, Nick Taylor ${ }^{1}$, \\ Nicole Krämer ${ }^{2}$, and Patrick Olivier ${ }^{1}$ \\ ${ }^{1}$ Culture Lab, Newcastle University, Newcastle upon Tyne, UK \\ ${ }^{2}$ Social Psychology: Media and Communication, University of Duisburg-Essen, Germany \\ \{rob.comber, anja.thieme, ashur, rafiev, nick.taylor, \\ patrick.olivier\}@ncl.ac.uk, nicole.kraemer@uni-due.de
}

\begin{abstract}
In this paper we continue work to investigate how we can engage young adults in behaviors of recycling and the prevention of food waste through social media and persuasive and ubiquitous computing systems. Our previous work with BinCam, a two-part design combining a system for the collection of waste-related behaviors with a Facebook application, suggested that although this ubiquitous system could raise awareness of recycling behavior, engagement with social media remained low. In this paper we reconsider our design in terms of engagement, examining both the theoretical and practical ways in which engagement can be designed for. This paper presents findings from a new user study exploring the re-design of the social media interface following this analysis. By incorporating elements of gamification, social support and improved data visualization, we contribute insights on the relative potential of these techniques to engage individuals across the lifespan of a system's deployment.
\end{abstract}

Keywords: Engagement, Facebook, Sustainability, Recycling, Gamification, Social influence, Persuasive technology.

\section{Introduction}

The disposal of waste is a mundane behavior. Being part of our daily routines, it does not request much of our attention. The impact of waste disposal on the environment however suggests that we should bring more awareness to our waste-related habits [35]. HCI has proposed a number of means through which we can motivate individuals, groups and society to engage in behavior change, including personal informatics [25], persuasion [14,16], gamification [7,23,22], social influence [5,27], and in small number of cases coercion $[32,15]$. Persuasive technology has become one of the key trends in this regard (see $[3,10]$ for a review of the sustainability literature). Yet, in direct opposition to the lack of motivation we experience on a daily basis, persuasive technology proposes that designers should focus their influential potential on those who are already motivated, facilitating their paths to goal fulfillment [14]. 
The question then arises of how we first entice people to be engaged in a process intended to change their behavior. In this paper we explore our continued research in this area with the BinCam system. The BinCam system is a two-part persuasive technology, with which we explore issues of engagement, persuasion and motivation for recycling and food waste behaviors. The system comprises a household landfill bin, fitted with a mobile phone in the lid, which captures images on closure and shares them on a dedicated Facebook application. Although the system aligns itself with sustainable HCI, our primary goal here is to understand and motivate engagement. In this light, we recognize that monitoring or engaging people in recycling and food waste behavior is not intrinsically motivating for most people. As such, we consider it a significant challenge for HCI, and society more generally, to contemplate the means through which we can engage individuals in such behaviors.

\subsection{Context and Motivation of the Research}

Engagement in recycling behavior is a significant challenge for individuals in the UK aged 18-35, who are largely unaware of the problems associated with inappropriate disposal of waste [35]. We previously explored this issue with regard to recycling and food waste behaviors from the perspective of habits [4] and as a problem of awareness and reflection [32]. In both cases we employed social media to facilitate engagement in behavior change. Research has shown the positive effects of including others in behavior change efforts [5,27] and related positive impacts of social media $[12,15,23]$. Social media provides a platform through which we can leverage a number of channels for behavior change, including personal informatics, social informational and normative influence [8], persuasive messaging and hedonic motivation.

Findings from our previous research revealed that, although individuals in the target group have strong positive attitudes towards recycling and sustainability, they often do not act towards these attitudes. While the data capturing part of our system served as a means to draw attention to waste disposal behavior, engagement with a social media application associated with the intervention was less successful. We noted particularly that individuals were not motivated to use the system beyond a short period of time. From our previous work, we are now faced with two questions: How can we further engage participants in discussion around recycling? How can we promote engagement with social media as a means to facilitate this discussion?

Driven by these questions, we explore the re-design of the social media During the re-design of the BinCam system, we have incorporated lessons learned from both the previous study, and through a critical analysis of how HCI understands and designs for engagement. We contribute an analysis of how engagement is understood in HCI; the strategies employed to support and promote engagement; an empirical analysis of engagement techniques in waste disposal behavior; and a critical reflection on engagement with, and use of, social media as a means to promote behavioral change.

\subsection{Engagement}

There is considerable diversity to how HCI and related disciplines have conceptualized engagement. There is also much confusion about what constitutes 
engagement, as it is often used interchangeably with notions of participation, immersion and attention. HCI has appropriated the concept of engagement ranging from the broad indicator of the quality of interaction to the cognitive measure of attention [29]. In the development of web applications, engagement is measured on at least three axes. As [24] put it: "Successful web applications are not just used, they are engaged with; users invest time, attention, and emotion into them." Such engagement is most often measured on two planes - first as behavioral engagement measured through behavioral data such as mouse clicks, time spent on pages and applications, and secondly as affective engagement, as captured by measures of satisfaction and affective response in questionnaires.

Engagement has also been understood in education and learning as the physical and psychological effort' devoted to a task [1]. Importantly, it is understood to have quantitative and qualitative features, to occur along a continuum and to impact on the effectiveness with which individuals achieve their goals. While physical effort pertains to behavioral engagement, psychological effort relates to a notion of cognitive engagement.

The exertion of psychological effort has been further considered in HCI research in terms of flow or optimal experiences [6]. Flow experiences occur when an individual is fully (emotionally, cognitively and physically) immersed in a task. Such immersion is not felt as requiring significant effort and is intrinsically motivating. Although flow represents optimal experience, it would not be expected to occur across a sustained and mundane activity, such as waste disposal. Flow experiences may also limit selfreflection, suggesting a non-conscious intrinsic engagement.

The recent move towards richer accounts of HCI has shifted the focus of engagement to understand it as the meaningful interactions an individual has with an artifact or service, and the quality of the attachment a user has to an object. While less easily quantified, such meaningful interaction has become a critical factor in third wave HCI $[2,28]$ and extends beyond affective engagement. Such work draws on an understanding of engagement as the meaningful and effortful reflection on activity in experience [9]. This reflective engagement involves the critical reflection on on-going activity, thinking over and through current actions as they occur.

There is also an increase in the application of social dynamics in the design of persuasive systems [27,32]. Such dynamics can impact on individuals' performance of behavior positively in increasing engagement (e.g., social facilitation), or negatively in decreasing engagement (e.g., social loafing). Thus, although it relies on a variety of interpersonal and personal factors, and incorporates elements of affective, cognitive and behavioral engagement, this can be collectively understood as social engagement.

Against the backdrop of this theoretical conception, the following presents a variety of strategies for engagement, commonly applied in persuasive HCI.

\subsection{Strategies for Engagement}

In attempting to promote engagement with online mental health interventions, Doherty et al. [11] suggest designing systems that are interactive, allow for personal 
experiences, provide support for the individual and facilitate social contact with others. Engagement strategies borrowed from the field of personal informatics [25] are mostly targeted at rewarding the user if the desirable behavior has been performed. Very little research in HCI has so far been dedicated to studying the effects of negative reinforcements to promote behavioral change [e.g., 15,23,32].

Interactivity. Interactivity relates to the providence of rich and varied experiences through the use of the system, which actively invite user exploration. This can, for instance, be achieved through diverse representations of peoples' behavioral data providing insights about their performances, whether they improved or how their behavior compares to others [11]. At its most basic, interactivity affords behavioral engagement; where the ability to perform actions and receive responses invites users to further engage with the system.

Space for Personal Appropriation. Personal experiences are often achieved through tailored designs based on the individual preferences of the user, which facilitates a sense of control as well as ownership [11]. Personal appropriation lends itself to affective engagement, where individuals perceive similarity to or ownership of an interactive system [33]. With personal appropriation, group identification could increase affective engagement through affording a sense of belonging, but also behavioral engagement through normative influences. That is, the presence of normative in-group behaviors may persuade individuals to engage in group-similar behaviors.

Behavioral Support and Reminders. In the context of health supporting interventions, the 'supportive' strategy is intended to improve peoples' adherence to a treatment program [11]. It is assumed that implemented (personal) support, such as a recycling coach or a recycling guide in our context, helps increase an individual's motivation to display or continue a certain behavior. This engagement strategy, however, is targeted at motivating behaviors that the individual is already familiar with, therefore only requiring support to be reminded of, or encouraged to perform the behavior [13]. The appropriation into a routine of behavior change appeals to a notion of behavioral engagement, but at the expense of cognitive engagement - where, as we have discussed elsewhere [4], the performance of behaviors becomes habitual.

Social Support and Social Media. Social engagement considers the importance of peer support to increase engagement with a system and to overcome motivational barriers to display a desirable behavior. It is therefore not surprising that social media sites like Facebook and Twitter have become increasingly popular platforms for the study of social support in the field of persuasive technologies [e.g., 21,22]. This engagement has massive potential for how we might design technologies for behavior change.

There is, however, also evidence suggesting that frequent use of social media is associated with a lower need for cognition [35]. Thus, although users of social media may have characteristics, such as a high need to belong to others, that make them 
more susceptible to persuasion, they may not be prone to persuasion through a direct route; that is, through the quality of information provided. Thus, research suggests that reflective and cognitive engagement are less likely to be associated with high levels of social media use.

Positive Reinforcement. Other strategies, often found with personal informatics systems [25], relate to how this data is fed back to the user. Most designs in this regard focus on strategies of positive reinforcement, presenting visual incentives to the user, to foster compliance with desirable behaviors. Such reinforcement drives affective engagement, which in turn may drive behavioral engagement. With UbiGreen, Fröhlich et al. [16] displayed a tree graphic to indicate green transportation activity, with the tree accumulating leaves, blossom or apples the more the individual uses environmentally friendly transportation. Persuasive designs in the field of sustainable HCI also commonly include visualizations of reduced energy consumption, carbon emissions or ecological footprints [17,20], or highlight money savings if the creation of waste is avoided [18].

Gamification and Achievements. In the context of behavioral change, gamification has been used to encourage positive behavior that the user would not normally engage in [30]. Gamification is defined as "using game design elements in non-game contexts to motivate and increase user activity and retention" [7]. Such engagement may tend towards intrinsic engagement, though this depends on how challenging and rewarding game elements might be. Gamification has been increasingly popular in both research and commercial systems. Design elements common to games, including scoreboards and badges, have been used to reward desirable activity [26], such as a regular use of an application.

Much like positive reinforcement, such game elements might increase affective engagement, where they are valued, indicate esteem or personal achievement. Even in professional contexts, the use of scoring systems has been demonstrated to increase use of an internal social networking site [34]. When combined with social networks that make these achievements visible, these features introduce an element of competition between, and playful awareness of others. For example, Foursquare (https://foursquare.com/) encourages users to check-in regularly by declaring the most active user in a location as 'mayor', but they can be replaced by another user if they fail to remain active. Thus, gamification also lends itself to social engagement, particularly in terms of competition and group identification. In these circumstances, a scoreboard can provide both a source of pride and a sense of shame when undesirable behavior is exposed.

Negative Reinforcement and Coercion. Far less research has examined the potential of negative reinforcements or coercion to promote behavioral change in HCI. Exceptions include research by Kirman et al. [23], who argue that behavioral change technologies should employ constructive aversive feedback alongside strategies for positive reinforcement to support the learning and maintenance of desired behaviors. Negative reinforcement in this context means that the performance of a behavior 
prevents or removes a negative response (e.g. a person may recycle to avoid disapproval by others). Engagement may be driven by avoidance of negative affect and through reflection on the actions that have led to negative outcomes. Foster et al. [15] have shown that a light form of coercion in the form of aversive feedback does not necessarily disengage users, as previously claimed by [5], but instead can function as a valuable component for achieving behavioral change. While ethical questions remain about the use of coercion, findings of this research revealed that aversive feedback can be a useful supplement in promoting behavioral change if designed carefully.

\section{Interface Re-design}

Following from the previous study with BinCam [4,32], a re-design of the system was undertaken following the potential and strategies to design for engagement and lessons learned from previous evaluations. Three strands of development were initiated, aiming to improve (1) system reliability (including $\mathrm{WiFi}$ and $3 \mathrm{G}$ connectivity); (2) feedback accuracy and frequency; and (3) overall engagement with the Facebook interface. Our focus here is on the third and final element, though the development of a more stable, reliable and trustworthy system contributed to a more robust experience for participants.

\subsection{Design for Engagement}

With the BinCam system, the Facebook application (short 'app') is the primary system front-end with the main goal to give users feedback on their recycling behavior and help them reflect on their own and other people's waste-related performances. Based on participants' experiences with the system we have suggested techniques to increase engagement with the Facebook application [32]. These included: a neat integration within the ecology of Facebook, challenges to promote group identification and competition on Facebook within and across different households, more frequent and varied visualizations for cognitive and reflective engagement, and improved opportunities to compare own waste-related achievements with other BinCam users. Below we position these within our framework for engagement and detail our strategies for redeveloping the app. The app offers a set of interactive elements to explore such as a BinLeague, including a variety of different visualizations of collected waste data, as well as creative BinProfiles of each BinCam bin in the system. BinAchievements are playful elements that can be gained through interactions with the interface, or engagements with specific recycling or food waste BinChallenges initiated by the BinMan.

BinMan. The BinMan is a virtual person on Facebook that is managed by an administrator of the BinCam system. The BinMan has a personal profile page on Facebook and personifies the BinCam system by posting recycling-related information on his wall, leaving comments, answering questions, and acting as a 
referee to the BinChallenges. The role of the BinMan is to improve the social component of the system and to facilitate users' social engagement with the system. As a social actor, he allows for the flexible and dynamic provision of support and knowledge, while simultaneously allowing for personalization and interactivity when responding to, or posting comments, thereby fitting into the ecology of Facebook.

BinLeague. The BinLeague was originally designed to give participants access to a record of the recycling activity of their household. Following poor precision in our previous studies using Amazon's Mechanical Turk, the tagging interface was redesigned to allow administrators access to the images and tag them for categories of landfill, recyclable, compost and food waste items. The BinLeague summarizes daily results for all bins in the system. Thus, it served as a personal informatics tool for reflective engagement and helped create a sense of in-group identification and outgroup competition for social engagement. The page provides a variety of different visualizations of the scores, extending the previous BinCam interface design. As in the original interface, each score has a unique visual representation, e.g. the recycling score is represented with a tree sapling that grows taller the better the score. Daily statistics reflecting the bin usage for a specific day are presented as a 24-hour graph, with each thrown away item producing an incremental progression on this graph.

BinProfiles. Additional bin statistics are also displayed on the bin's profile page and contain information on the daily bin usage, graphing the number of items in the bin according to the four tagging categories outlined above. As an additional playful team-building experience, each household has been asked to choose a profile picture for their bin from a set of 18 images. Allowing for personalization and affective engagement, the profile picture personifies the system, so the BinCam becomes a mascot of each household. The start page of the BinCam app displays the list of bin profiles and showcases awards given for succeeding in the BinLeague and the BinChallenges.

BinChallenges. BinChallenges are managed manually by the BinCam administrator and delivered through the BinMan's news feed. The purpose of the challenges is to boost user interest when needed, by providing activities that might be intrinsically engaging, or which promote certain waste-related actions. Most of the challenges required participants to respond by being creative in using the system, for example: "The funniest message on non-recyclable waste wins". By promoting competition between households it might also be possible to increase social engagement with the system.

BinAchievements. The system of achievements defines a number of fixed goals for the user. It is aimed to increase user engagement with the system and to encourage more exploration of the interface. The achievement system is automatically administered and gives an immediate feedback to the user. All achievements can be divided into three major groups: regularly visiting the system, browsing images and leaving comments. 


\section{Evaluation}

For a period of six weeks we deployed one BinCam bin in a total of six student houses in Newcastle upon Tyne, UK. Prior to the start of the study all members of the household were introduced to the system and completed a pre-study questionnaire on their food waste and recycling attitudes and behaviors, and the Facebook Intensity scale [12]. The Facebook Intensity scale is a measure of Facebook use, including measures of behavioral and emotional engagement. Example items include for instance "Facebook has become part of my daily routine" or "I feel out of touch when I haven't logged onto Facebook for a while". Following the study, participants were invited to either a focus group or an individual interview and completed a postquestionnaire. One member of each household was randomly selected for an individual interview and the remaining members took part in a focus group. In total, five participants completed individual interviews, with only one invited individual not responding. The six focus groups involved 27 of the participants, with one invited participant not attending. The individual interviews were carried out to gain a sense of how individuals perceived the system and particularly to consider how individual concerns might differ from those expressed in the group. Focus groups also allowed for the consideration of group dynamics and for the exploration of social and normative influences. In both conditions, participants related similar concerns. In the data presented below all names have been changed.

\subsection{Participants}

34 individuals completed the pre- and post- questionnaire for the study. The study sample was aged 18-27 ( $\dot{\mathrm{X}}=21.12$, sd.=1.93), of whom 20 were female. Two participants were in part-time education, with the remainder in full-time education. 17 were in the first year of third level education, three in the third year of an undergraduate degree, 12 were enrolled in Masters level education, and one undertaking a $\mathrm{PhD}$. One student was an exchange student from an international university. All but one flat had students at different stages of education. Five households had 6 participants and one household had 5 participants. Three households were mixed gendered ( 3 female, 3 male) and three households were single gendered (one household of 5 males and two households of 6 females).

\section{$4 \quad$ Results}

\subsection{Recycling and Food Waste Attitudes}

As with our previous study, pre- and post- questionnaires revealed little change in participants' attitudes to recycling and food waste. Participants, partially due to selfselection and social desirability, report strong positive attitudes towards sustainability from the outset. This leads us to reiterate our previous assertions that, within rational choice models, recycling and food waste might be better motivated by examining 
issues of awareness and perceived behavioral control. There were some significant changes in social aspects of recycling. Most participants for instance reported changes in the social aspects of waste disposal (e.g. "I ask other people for advice as to how I can keep food for longer"), waste disposal knowledge (e.g. "I think food waste is difficult to avoid") and in feelings associated with waste disposal (e.g. "I recycle because I feel better if I do").

\subsection{Facebook Use}

On average the participants had 449 friends and spent 90 minutes a day on Facebook in the week prior to deployment. The average Facebook intensity (FI) score for the sample was $3.52(\mathrm{n}=31$, $\min =.91$, $\max =4.93)$, suggesting that the participants are above average Facebook users. Comparing changes in pre- and post-questionnaire items further suggests that Facebook use is inversely related to commitment to change $(\mathrm{r}=-.438, \mathrm{n}=30, \mathrm{sig}=.016)$ and contemplation of changing behavior $(\mathrm{r}=-.437, \mathrm{n}=30$, sig=.016).

In order to more closely examine possible relationships, Facebook intensity was also correlated with responses to recycling behavior. Questions correlating FI with recycling predominantly related to social concerns (e.g. "I listen to what my flat mates have to say", "I ask other people for advice as to how I can keep food for longer") and identity performance (e.g. "I try to conceal food waste that I dispose of"). FI was negatively correlated with concerns about the cost of food waste (e.g. "Throwing away food costs me money" and "I buy fresh food on special offer like buy one get one free or three for two") and general concerns about food waste ("throwing away food bothers me"). FI also positively correlated with beliefs about local facilities (e.g. "We have adequate facilities in our local area to recycle"). In each case the correlations were moderate and although they generally point towards the findings of [35] no strong relationship between FI and recycling attitude is supported in this study.
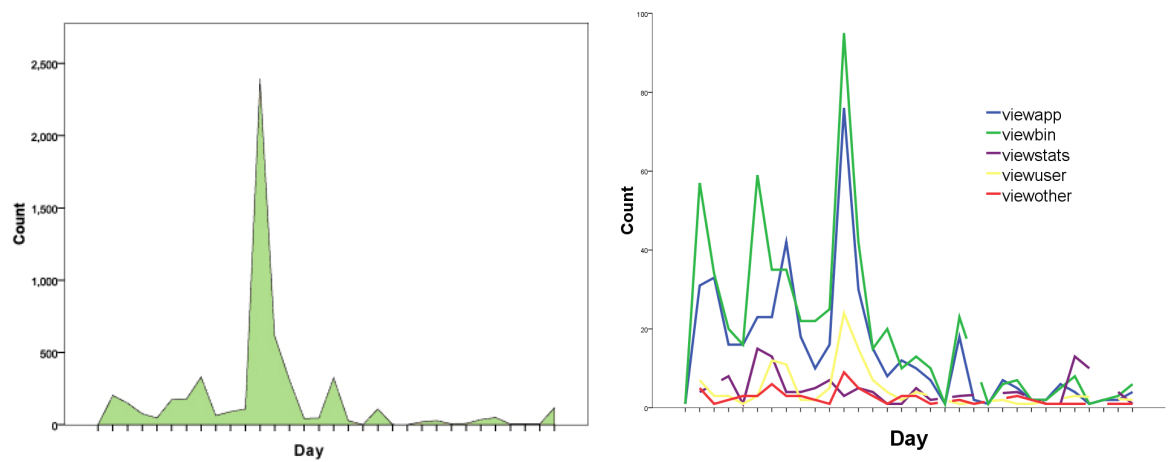

Fig. 1. \& 2. Total daily activity (left) and daily levels for each activity, excluding viewing images (right). A large increase surrounds one user's action 2 weeks into the project. Following this, there is a continued drop off in activity, with only minor increases coinciding with BinChallenges and activities at the end of the project. 


\subsection{Using the Interface}

Of the 32 participants to complete the study, seven did not log into the Facebook interface. Behavioral engagement metrics for the remaining 25 participants were gathered from the BinCam application for a range of activities (see total activity in Figure 1). These were (with total number of actions, and percentage of overall activity): view application title page $(444,7.9 \%)$, view BinProfile $(598,10.7 \%)$, view BinPictures $(4277,76.3 \%)$, view user $(115,2.1 \%)$, view BinLeague $(83,1.5 \%)$, view daily statistics $(22,0.4 \%)$, view FAQ $(12,0.2 \%)$, and view BinChallenges $(55,1 \%)$. There were no significant relationships between scores on the FI scale and the use of the application or specific aspects of the system. There were no significant differences in access to or use of the system between households. Viewing BinPictures is a significant part of the overall activity. One individual, however, is responsible for 2124 image views, constituting $37.89 \%$ of all activity. This occurred predominantly in one sitting. During an interview he explained that he was motivated to do so to both gain achievements and to compete against another participant in a different household who had gained more achievement points than him. When figures for image viewing are removed, the application title page and BinProfile contribute over $78 \%$ of the activity ( 33.4 and $45 \%$ respectively). This suggests that the use of the system is somewhat limited to these features.

The majority of interaction occurs within the first two weeks with a peak in the second week. The peak of activity occurs as the participants become familiar with the application, after most participants have logged in, and as they begin to discuss it among themselves. Shortly afterwards, the level of activity drops off. In order to better understand the activity patterns, we can examine the daily activities, excluding image viewing (see Figure 2). Sophie summarizes this use pattern:

"Erm... I would say... I logged in quite a bit in the beginning of the project... and then just as the weeks gone on I didn't bother anymore.... erm... I say... when one of the challenges went up...that made me log on again..."

An analysis of variance and post-hoc tests suggest that the activity of only the topmost quartile was significantly different from the others for views on BinProfiles (F: 24.35, sig: .000), posting comments (F:8.34, sig: .001), visit application title page (F:39.83, sig: .000), view user profiles (F:8.28, sig: .001), and other activities such as viewing the FAQ (F:6.08, sig: .003). This suggests that a small number of users contribute significantly to the overall activity on the application and might be considered engaged with the system.

\subsection{BinAchievements}

A number of users were motivated to engage in searching for and gaining BinAchievements. Achievements were awarded for the engagement with the app in three categories: logging in, commenting and viewing images. In each case, achievements were incremented through progressively more engagement (e.g. view 1 picture, view 10 pictures, etc.). All participants who logged into the application 
received a BinAchievement for doing so. Users of the site received on average 4 achievements, or 58 achievement points, beyond the first achievement which was for logging in. There was no significant correlation between FI and achievements received.

The majority of BinAchievements were awarded in the first two weeks and only a small number of participants were motivated to gain achievements mid-way into the project. One user who was motivated to gain all achievements did so across one session. In general, however, the achievement trajectories suggest that only a small number of users were reminded of or engaged with achievements on the BinCam Facebook app in the $3^{\text {rd }}$ and $4^{\text {th }}$ weeks of the study. Brief resurgence of interest appears to be associated with a social BinChallenge and later the closing of the system.

\section{$5 \quad$ Results from the Interviews and Focus Groups}

The quantitative use data suggests that there was no sustained engagement with the Facebook application, but that some users experience intense engagement at the start of the study. We therefore look to qualitative interview and focus group data to understand why this might be the case and how, if at all, individuals had engaged with the system. Although our primary focus is on the social media interface, the ubiquitous system figures as a concern in how users' engage with the overall system.

\subsection{Awareness, Guilt and Perceived Behavioral Control}

Like in our previous work, significant findings from this study point to improved awareness among participants. In this study we gain a clearer understanding of how this occurs and how the ubiquitous system draws users to engage with their food waste and recycling behaviors. Specifically, we gain insight into the transition from under-awareness to mere awareness to engagement.

From Awareness to Routine. Participants are drawn to attend to the bin by its novelty, the awareness of it taking pictures, and the shutter sound of the camera when a picture is being taken, summarized by Eva:

"Yeah, I think I was more aware as well cause like with it making the noise I think...It was the vibrating I think and also like... I don't know just because it's a different bin to what we have before. It was obvious like that gonna be uploaded."

As the presence of the BinCam bin leads to raised awareness, this awareness led to personal motivation to change:

Mary: “... [the BinCam bin] makes you more aware and kind of want to do it right..."

Participants experienced affective engagement with the system, feeling it morally correct to change their behavior. As the novelty of the system recedes over time, 
participants begin to lose some awareness of it as a persuasive system and therefore have diminished cognitive engagement with it. The bin itself though remains cognitively demarcated from the recycling bin:

Sophie: "Erm... it kinda for one second got on my mind that bin was only stood for landfill kinda thing. It didn't... like I didn't think: "Oh it's going to take the picture now." I wasn't like listening out for the sound every time...erm....but erm at the beginning I was always thinking: "Right... ok... consciousness decision which bin am I gonna use..."."

Thus, participants think of the BinCam as fundamentally different, and for landfill waste only and not as a recycling bin. Consequently, their engagement with the bin is behaviorally and cognitively different. This becomes a practiced routine and relatively unconscious knowledge. Further exploration reveals that this transformation from awareness to engagement may be both the forming of a habit and the avoidance of negative affect.

In our previous study, participants' sense of the system as one for behavior change had led to feelings of guilt, primarily about differences between attitudes and actual behavior. In this study, the drive to 'do it right' still led some participants to feelings of guilt. It became clearer in this study, however, that participants differentiate this guilt from feelings of shame:

Tom: “...I did feel guilted in to doing sometimes but I never felt ashamed because my guilt preceded the shame."

Feelings of guilt that arose were induced by personal reflection on behavior and motivated individuals to change behavior to reduce guilt. Thus, it is clear that the BinCam system, at least for some participants, promoted reflective engagement that resulted in negatively experienced affective engagement.

Persistent Awareness. One area where this diffusion of awareness did not occur was in the case of food waste. In all cases, the participants either did not have access to composting facilities, or would not be able to use compost. Consequently, although they felt guilty about food waste, there was little they could do:

Neil: "I think we are really good at recycling in our house but in terms of food waste we are probably not so but... I think that's more with the university aren't providing... a compost bin."

Thus, at every occasion where food waste was to be put in the BinCam bin, participants' attention was drawn to the behavior. Participants, who felt strongly about food waste found ways to adjust to minimize feelings of guilt or heightened awareness of inappropriate behaviors:

Sophie: "it's made me just kind of just reduce my portion size and then think about how much stuff I'm throwing away and trying to catch things before they go out of date and stuff like that." 
These new strategies are likely to reduce engagement with the bin while increasing engagement in positive food waste behaviors. Furthermore, while this might reduce overall interaction and awareness of the system, it is likely to decrease the likelihood that the system's presence in food waste behaviors becomes routine. That is, the presence of the BinCam bin continued to draw attention to itself in food waste behaviors.

\subsection{Gamification}

With the participants we wanted to further explore their experiences with gamification. The achievements were designed to be discoverable, and the app provided information on how achievements could be received. For most of the participants, gaining achievements was first unintentional, following which they were motivated to find more. However, the value of the achievements for motivation quickly reduced, particularly where the activity involved was repetitive:

Peter: "... erm... I got the achievement that were easy-ish and it took me a few minutes I guess. Erm and then some achievements like you said like the 250 [viewing pictures] it was just like the same as the 50 one but just more...It's kind of... cause it's just like a repeat of the same I guess I just thought... [it is not worth it]"

For others, despite initial excitement, there was no further motivation to engage until after the study:

Clare: "I wanted to get more.

Jill: Yeah. I should have gone back to get more.

Clare: And maybe log on more."

For some participants, the design for gamification of recycling was motivating, and, as previously mentioned, one participant was driven to contribute almost $40 \%$ of all activity by wanting to compete with someone else. However, for most users this was not the case and many did not feel they might ever be engaged with such an activity:

Jayne: "I didn't really... I wasn't really interested in looking at what's in my own bin [...] let alone what's in other people's bin... or playing like "inter-bin-related games",".

The challenges which drew most interest were those that involved some aspect of household team work, such as leaving a funny message in the bin or taking a picture of the group with the bin. Such challenges were appreciated by most participants, and they were among the only images to elicit cross-household activity:

Jayne: "[...] We looked at pictures of other people's challenge... that joke challenge... we looked at that... We didn't look at pictures of people's like... 'crap in the bin"”

\subsection{Facebook Ecology and Daily Routines}

The decision to design for Facebook was based on its proliferation as a social and engaging platform. We had also, from our previous work [32], highlighted that the 
system must more closely align with the existing Facebook ecology. The behavioral engagement demonstrated, however, suggests that the application did not harness the benefit of being associated with Facebook. This appears to have been due to three difficulties in assimilating into the ecology of Facebook. First, some users simply do not use Facebook frequently. This prevented them from engaging with the BinCam app entirely. Second, some users did not use Facebook apps frequently.

Bill: "I know... I well, to be honest I don't really use Facebook that much and I've never... I don't think I have ever actually use an app on Facebook or anything else".

Finally, the configuration as an app impeded the extent to which messages from the BinMan were shared with users. Furthermore, most users suggested that the BinMan could post with higher frequency - on the one hand to increase visibility, and on the other to increase the amount of interaction between users and the system.

Although BinCam is designed to be situated within everyday practices in student households, some of the practices and routines of student households also decrease the possibility for social support and discussion around the system. In one household, the participants reflected on their routines, in saying:

Sam: “... it's rare that we are all in together...I see you like once in three days...".

Peter: "[laughing] Same here. We cook in different times and stuff as well usually... so there wasn't really mentioned of it [BinCam]."

\section{Discussion}

Users' engagement with the BinCam bin and Facebook application revealed mixed effects. The main impact of the BinCam system continues to be in raising awareness of recycling and food waste behavior. The audio cue from the bin serves as a reminder throughout their engagement, and as previously noted [4], acts as a postactional cue for reflection. Thus the system draws attention to itself, which raises reflective engagement in the individual. This brought about a change in participants' behaviors where they reduced the amount of waste they produced.

The fact that some people do not engage with online and competitive games is not a new finding. The motivation to engage individuals with competitive game elements is, among other things, gender differentiated [19]. In critical literature on gamification [7], there is an assertion that gamification must mean more than simply awarding points and badges and showing these on leader boards. The empirical data on the use of BinCam suggests that this is the case. Although we were able to achieve two weeks of engaged use, there was little further use of the application. If we understand a 'game' as something in which we are challenged and must overcome challenges [7], then perhaps recycling is not such an activity. We have however focused on engagement with the system and not recycling itself.

The integration into everyday practices of the social media platform was not always successful. As stated, the use of Facebook and Facebook apps was not always within the routines of users' everyday behavior. In the case of waste disposal, simply being subsumed into everyday practice, particularly when those practices are habitual, 
means that it is difficult to create awareness or to change behavior. From this study we have examples of how designing to disrupt everyday practice both worked and did not work to create engagement. In the case of the audio cue from the bin, this was sufficient to disrupt the routine of waste disposal. While the post-behavioral audio cue did not change behavior in the moment, it created reflective engagement as it drew attention to the unconscious performance of it.

BinPictures were described as unappealing and lacking interest. Despite this, they received far more activity than any other aspect of the system - even when excluding extreme users. This is at least partly due to the influence of achievements and gamification. Thus, despite being potentially uninteresting, BinPictures had the most appeal as an interaction. Moreover, the mundane and particular nature of waste disposal meant that most individuals were not interested in viewing images of waste. There was little evidence here of either intrinsic or affective engagement. There was no real added value in seeing pictures from the bin, because people didn't care about them.

However, social challenges did increase participation, and many participants reported these challenges to be the most enjoyable aspect of the study. This is in line with [19] that games including meaningful social interaction can increase appeal. And although few participants sought support through the system, many participants reported discussing recycling issues within their household. This appears to be particularly important for the acquisition of recycling knowledge. Moreover, such sharing may expand the cultural knowledge [31] that underpins individuals' recycling knowledge and provides the means and skills to adapt to, for instance, new expectations about what can or cannot be recycled. It is therefore critical that research continues to explore the specific mechanisms through which competitive and noncompetitive social engagement can be fostered in interactive systems for behavior change.

The use of the BinMan as a conduit for information on Facebook did not disrupt participants. In fact, several wished for more feedback and notifications from the BinMan. With the use patterns for Facebook, where participants logged in occasionally, comments posted could be easily overlooked and were arguably not of a high enough frequency. This is not to suggest that bombarding participants with messages will achieve better engagement, but that such interventions should be tailored to the practices and expectations of participants. More visible or direct notifications outside of the Facebook ecology might be more effective (e.g. e-mail, SMS messages, or a shared, open visualization in the home and near the bin). Thus the ways in which persuasive technologies explicitly draw attention to themselves needs consideration.

\section{Conclusion}

This paper presented a user study of our re-design of the BinCam interface on Facebook. Although most participants use Facebook (and other social media) they do so in particular ways, at particular times, and fit these activities around their everyday routines. The social and material practices that are shared among households do not 
directly, or necessarily, involve social media. This varied between households, some were more engaged than others, but across almost all of them, the use of the BinCam Facebook application was largely an isolated and lone activity, as were the activities of recycling and food waste.

When we review this data we are left with, among others, a recurring question: is recycling and food waste simply so uninteresting that we cannot get people to engage with it in the long term? We do not believe that this is the case, and we consider our research with the BinCam system to present some progress in this regard. Significant challenges of course still remain. In particular, we have highlighted the necessity for integration of multiple forms of engagement and feedback into everyday life as a central concern.

Acknowledgements. We would like to thank all our participants, Mat Kipling, and Gu-Young Kang. This project has been supported through the European FP7 Marie Curie Action Balance@Home project and the RCUK Digital Economy Research Hub SiDE: Social Inclusion through the Digital Economy.

\section{References}

1. Astin, A.: Student involvement: a developmental theory for higher education. Journal of College Student Personnel 25(4), 297-308 (1984)

2. Bødker, S.: When second wave HCI meets third wave challenges. In: Proc. NordicCHI 2006, pp. 1-8. ACM Press (2006)

3. Brynjarsdottir, H., et al.: Sustainably unpersuaded: how persuasion narrows our vision of sustainability. In: Proc. CHI 2012, pp. 947-956. ACM Press (2012)

4. Comber, R., Thieme, A.: Designing beyond habit: opening space for improved recycling and food waste behaviors through processes of persuasion, social influence and aversive affect. Personal and Ubiquitous Computing (online first)

5. Consolvo, S., McDonald, D.W., Landay, J.A.: Theory-driven design strategies for technologies that support behavior change in everyday life. In: Proc. CHI 2009, pp. 405414. ACM Press (2009)

6. Csikszentmihalyi, M.: Finding Flow: The Psychology Of Engagement With Everyday Life. Basic Books (1998)

7. Deterding, S., et al.: From game design elements to gamefulness: defining "gamification". In: Proc. MindTrek 2011, pp. 9-15. ACM Press (2011)

8. Deutsch, M., Gerard, H.G.: A study of normative and informational social influence upon individual judgment. Journal of Abnormal and Social Psychology 51, 629-636 (1955)

9. Dewey, J.: How we think. Dover Publications (1997)

10. DiSalvo, C., Sengers, P., Brynjarsdóttir, H.: Mapping the landscape of sustainable HCI. In: Proc. CHI 2010, pp. 1975-1984. ACM Press (1984)

11. Doherty, G., Colye, D., Sharry, J.: Engagement with online mental health interventions: An exploratory study of a treatment for depression. In: Proc. CHI 2012, pp. 1421-1430. ACM Press (2012)

12. Ellison, N.B., Steinfield, C., Lampe, C.: The benefits of Facebook "friends:" Social capital and college students' use of online social network sites. Journal of Computer Mediated Communication 12, 1143-1168 (2007)

13. Fogg, B.J.: A behavioral model for persuasive design. In: Proc. Persuasive 2006 (2006) 
14. Fogg, B.J.: Persuasive Technology: Using Computers to Change What We Think and Do. Morgan Kaufmann (2003)

15. Foster, D., et al.: Wattsup?: motivating reductions in domestic energy consumption using social networks. In: Proc. NordiCHI 2010, pp. 178-187 (2010)

16. Fröhlich, J., et al.: UbiGreen: Investigating a mobile tool for tracking and supporting green transportation habits. In: Proc. CHI 2009, pp. 1043-1052. ACM Press (2009)

17. Fröhlich, J., Findlater, L., Landay, J.: The Design of Eco-Feedback Technology. In: Proc. CHI 2010, pp. 1999-2008. ACM Press (2008)

18. Gartland, A., Piasek, P.: Weigh your waste: A sustainable way to reduce waste. In: Ext. Abst. CHI 2009, pp. 2853-2858. ACM Press (2009)

19. Hartmann, T., Klimmt, C.: Gender and Computer Games: Exploring Females' Dislikes. Journal of Computer-Mediated Communication 11(4), 910-931 (2006)

20. Holmes, T.: Eco-visualization: Combining art and technology to reduce energy consumption. In: Proc. C\&C, pp. 153-162 (2007)

21. Jamison-Powell, S., et al.: I can't get no sleep": discussing \#insomnia on twitter. In: Proc. CHI 2012, pp. 1501-1510. ACM Press (2012)

22. Jianqiang, D.S., et al.: Farmer's Tale: A facebook game to promote volunteerism. In: Proc. CHI 2011, pp. 581-584. ACM Press (2011)

23. Kirman, B., et al.: Improving social game engagement on facebook through enhanced socio-contextual information. In: Proc. CHI 2010, pp. 1753-1756 (2010)

24. Lehmann, J., Lalmas, M., Yom-Tov, E., Dupret, G.: Models of user engagement. In: Masthoff, J., Mobasher, B., Desmarais, M.C., Nkambou, R. (eds.) UMAP 2012. LNCS, vol. 7379, pp. 164-175. Springer, Heidelberg (2012)

25. Li, I., Dey, A.K., Forlizzi, J.A.: Stage-Based Model of Personal Informatics Systems. In: Proc. CHI 2010, pp. 557-566. ACM Press (2010)

26. Liu, Y., Alexandrova, T., Nakajima, T.: Gamifying intelligent environments. In: Proc. UbiMUI 2011, pp. 7-12. ACM Press (2011)

27. Mankoff, J.C., Matthews, D., Fussell, S.R., Johnson, M.: Leveraging social networks to motivate individuals to reduce their ecological footprints. In: Proc. of HICSS, pp. 1-10 (2007)

28. McCarthy, J., Wright, P., Wallace, J.: The experience of enchantment in human computer interaction. Personal and Ubiquitous Computing 10, 369-378 (2006)

29. Peters, C., Castellano, G., de Freitas, S.: An exploration of user engagement in HCI. Proc. AFFINE 9(3) (2009)

30. Shiraishi, M., et al.: Using individual, social and economic persuasion techniques to reduce CO2 emissions in a family setting. In: Proc. Persuasive 2009 (2009)

31. Strengers, Y.: Designing eco-feedback systems for everyday life. In: Proc. CHI 2011, pp. 2135-2144 (2011)

32. Thieme, A., Comber, R., et al.: "We've bin watching you.": designing for reflection and social persuasion to promote sustainable lifestyles. In: Proc. CHI 2012, pp. 2337-2247. ACM Press (2012)

33. Thieme, A., et al.: Lovers' box: Designing for reflection within romantic relationships. International Journal of Human Computer Studies 69(5), 283-297 (2011)

34. Thom, J., Millen, D., DiMicco, J.: Removing gamification from an enterprise SNS. In: Proc. CSCW 2012, pp. 1067-1070. ACM Press (2012)

35. WRAP. Waste and resources action programme, http://www.wrap.org.uk (accessed December 29, 2010)

36. Zhong, B., Hardin, M., Sun, T.: Less effortful thinking leads to more social networking? The associations between the use of social network sites and personality traits. Computers in Human Behavior 27(3), 1265-1271 (2011) 